\section{Elastic Material with Systems of Growing or Closing Cracks: Tangential Stiffness}

\author{
Romesh C. Batra (Ed.)
}

\section{Contemporary Research in Engineering Science}

With 304 Figures

\author{
Z.P. Bažant ${ }^{1}$ and P.C. Prat $^{2}$ \\ ${ }^{1}$ Northwestern University, Evanston, IL 60208 \\ 2 Technical University of Catalunya, Barcelona, Spain
}

\begin{abstract}
The problem of calculating the overall elastic moduli of a microcracked material is now understood relatively well, however, only under the assumption that the cracks are fixed, that is, neither grow nor shorten during loading. Such a calculation yields the secant moduli for the response stress-strain curves of microcracked materials. The present paper shows how this existing knowledge can be extended to calculate the tangential moduli for incremental deformations of the material during which the cracks are allowed to grow and remain critical, or shorten. For this purpose, the conditions that the energy release rate of several families of cracks in the material must on the average be in balance with the energy dissipation rate characterized by the fracture energy of the material are formulated. The results of preliminary numerical studies still in progress are reported. It is found that the self-consistent scheme for the calculation of elastic constants of a microcracked material does not give realistic results except for deformations up to shortly after the peak load. On the other hand, the differential scheme yields realistic stress-strain curves and it also gives a ratio of uniaxial tensile to compression strengths that is approximately correct for initially highly microcracked materials such as concrete. A comprehensive report on the computation of tangent moduli will be given in a separate paper.
\end{abstract}

\section{INTRODUCTION}

Prediction of the response of structures made of damaging materials requires modeling of the effect of microcracks on the constitutive law. The basic problem is the effect of a random crack system, statistically uniform in space, on the elastic constants of the material. The spatial orientation of the cracks within the system may be assumed to be sufficiently random and statistically uniform in space to render the body isotropic or, on the contrary, it may be assumed that the cracks are arbitrarily distributed, with a certain number of crack families oriented along some particular directions, thus rendering the body globally anisotropic. For both types of crack distributions, the effect on the overall elastic properties has been studied extensively, and many important results have been achieved in fields ranging from plain and high-performance concrete to soil and rock mechanics and geophysical research [1-15]. However, the existing results are limited to elastic solids in which the cracks are stationary, that is, neither grow nor shorten. The objective of the present paper is to formulate the problem of response of materials in which 
the cracks can grow or shorten during loading. The method of solution will be proposed and some results from a numerical study in progress will be reported. A comprehensive numerical study will be left to a subsequent paper [16].

\section{LIMITATIONS OF CURRENT KNOWLEDGE AND EXTENSIONS NEEDED}

\subsection{Problem 1. Effective Secant Moduli}

During the last two decades, calculation of the stiffness tensor of a material intersected by various types of random crack systems statistically uniform in space has been systematically explored. Effective methods of calculation of the elastic moduli of such materials have been developed, based on application of various methods for composite materials, such as the self consistent scheme and the differential scheme [1, 17, 18]. Crack arrays of various configurations have been analyzed. Variational bounds on the effective elastic moduli of crack materials have also been obtained $[8,19]$. Highly accurate numerical solutions for various examples of bodies with various examples of specific crack configurations have been presented [8].

There is, however, one serious limitation of the current knowledge. All the studies so far have dealt with fixed cracks, that is cracks that neither propagate nor shorten (Fig. 1). This means that, in the context of response of a material with growing damage illustrated by the curve in Fig. 1, the existing formulations predict only the secant elastic moduli (such as $E_{s}$ in Fig. 1). Such information does not suffice for calculating the response of a body with progressing damage due to cracking.

\subsection{Problem 2. Effective Tangential Moduli}

To calculate the response of a material with cracks that can grow or shorten, it is also necessary to determine the tangential moduli, exemplified by $E_{t}$ in Fig. 2. Knowledge of such moduli makes it possible, for a given strain increment, to determine the inelastic stress drop $\Delta \sigma_{c r}$ (Fig. 2). This is obviously a harder problem than the calculation of the secant moduli, because additional conditions must be introduced to express the fact that the growing cracks must remain critical, i.e., their energy release rate must remain equal to fracture energy $G_{f}$ of the material, and that, for shortening cracks in which the crack faces are coming into contact, the energy release rate must be 0 . The method to deal with this problem will be addressed here under the assumption that, despite the growth of the cracks, their statistical distribution in space remains uniform. In other words, it will be assumed that the cracks do not localize into bands or some other domains. In that case, the material properties on a uniformly deforming specimen can be regarded as local material properties, and so the calculation yields the local effective moduli.

\subsection{Problem $\overline{ }$ 3. Tangential Stiffness of Material with Localizing Cracks}

Knowledge of the secant and tangential moduli (Problems 1 and 2) is still not sufficient to predict the response of a structure with growing cracks. It is now well known that softening damage caused by cracking tends to localize into cracking bands or other regions. The localization of cracking is caused and governed mainly by interactions among propagating cracks. The interactions cause that the average behavior of a representative
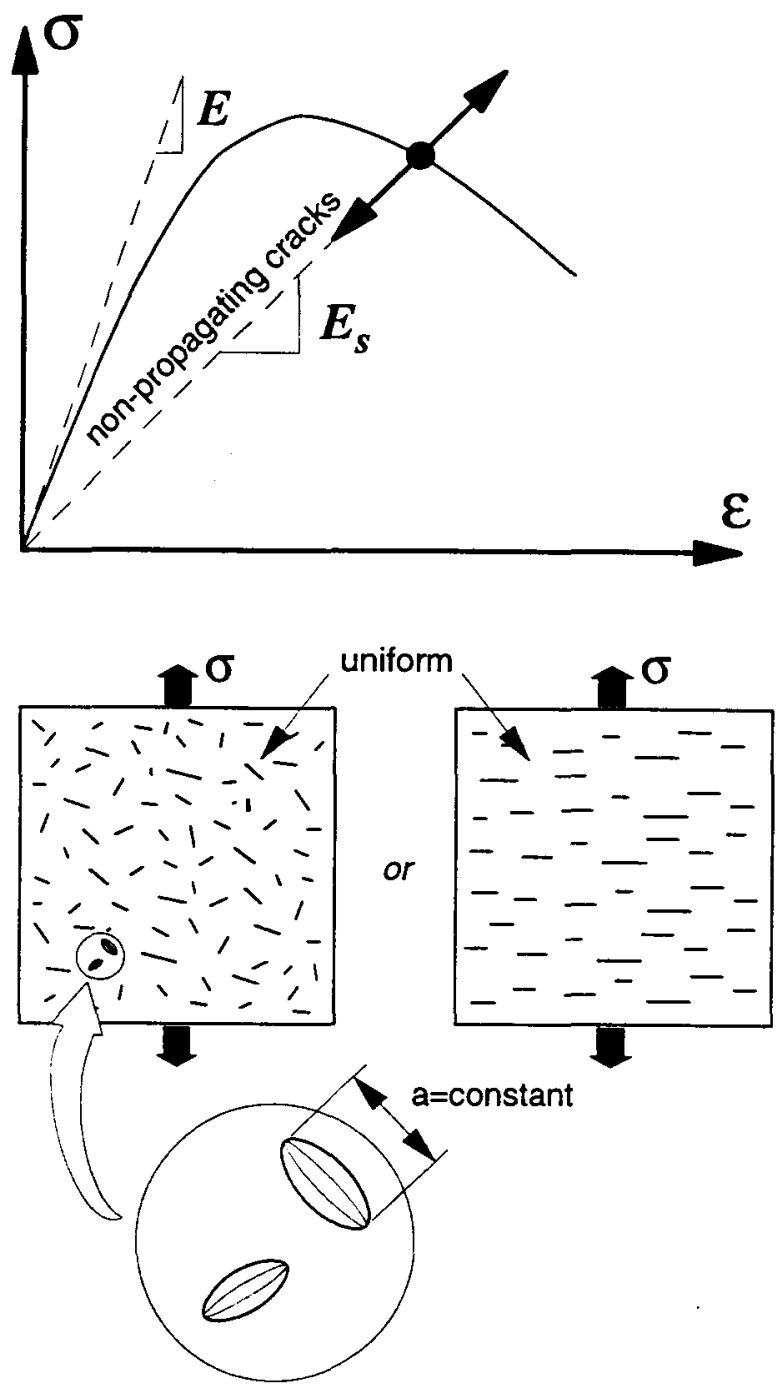

Figure 1. Effective Secant Moduli.

volume of the material with cracks does not follow the local stress-strain curve for growing cracks but follows a slope that is either smaller or larger, as shown in Fig. 3. This problem has recently been analyzed and an integral equation in space governing the nonlocal behavior of such material has been formulated [20-22] on the basis of smoothing of crack interactions. A complete solution of this problem obviously requires as input the information about the secant and tangential moduli from (Problems 1 and 2). Discussion of Problem 3 is beyond the scope of the present paper but will need to receive attention in the future. 

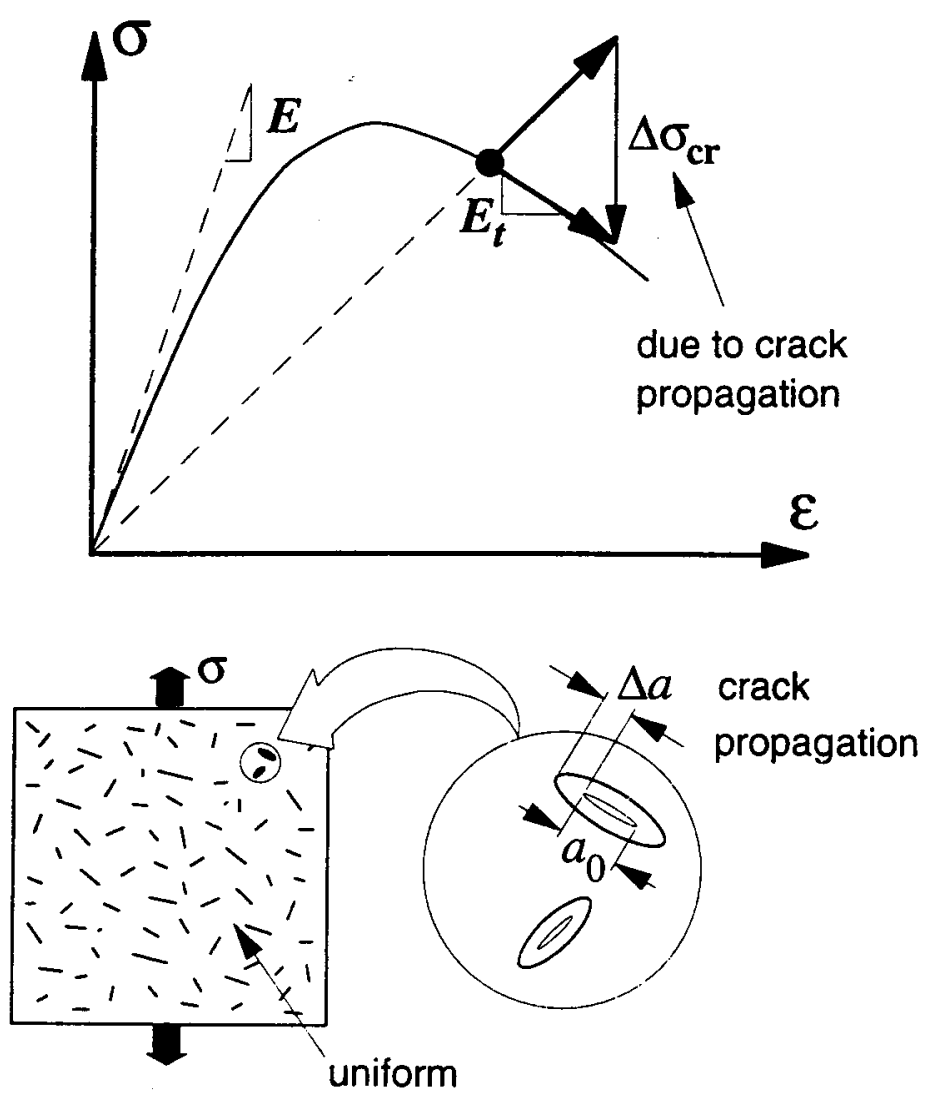

Figure 2. Effective Secant Moduli.

\section{METHOD OF CALCULATION OF TANGENTIAL COMPLIANCE TENSOR}

In terms of the secant compliance tensor $\mathbf{C}$, the constitutive relation of an elastic material with many random cracks that are statistically uniform in space may be written as

$$
\epsilon=\mathbf{C}: \sigma
$$

in which $\epsilon, \boldsymbol{\sigma}=$ macroscopic (average) strain and stress tensor, $\mathbf{C}=$ fourth-rank tensor, and the colon denotes a doubly contracted tensor product.

Consider now that the elastic body is intersected by $N$ families of random cracks, labeled by subscripts $\mu=1,2, \ldots, N$. Each crack family may be characterized by the average crack radius $a_{\mu}$, and the number of cracks per unit volume of the material in each family may be denoted as $n_{\mu}$. All the cracks may approximately be considered as
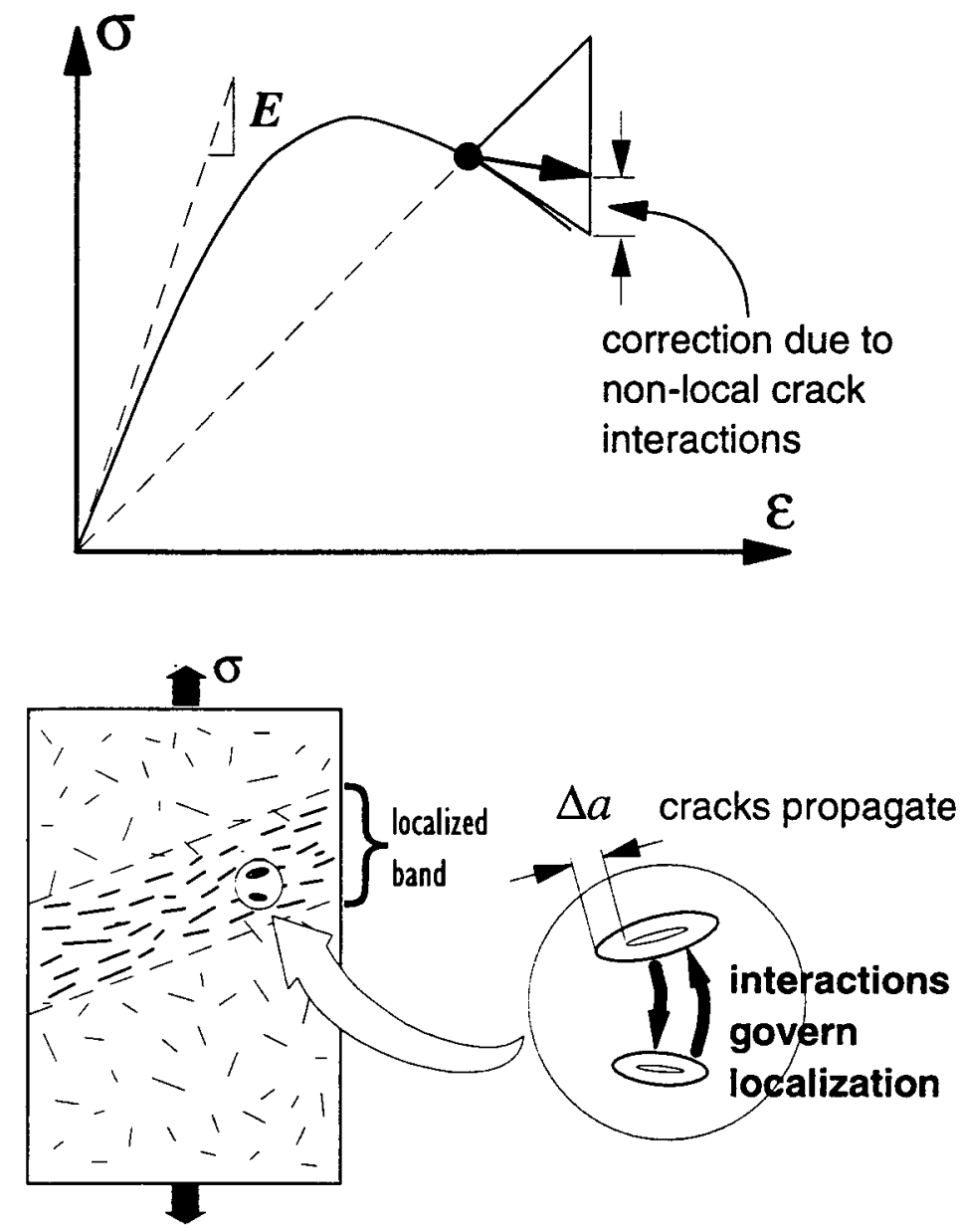

Figure 3. Tangential stiffness of material with localizing cracks.

circular, with radius $a_{\mu}$ in family $\mu$. Thus, the compliance tensor may be considered as the function:

$$
\mathbf{C}=\mathbf{C}\left(a_{1}, a_{2}, \ldots, a_{n} ; n_{1}, n_{2}, \ldots, n_{N}\right)
$$

Approximate estimation of this function has been extensively reviewed by Kachanov and co-workers $[8,19,23,24]$.

The incremental constitutive law can be obtained by differentiation of Eq. 1, which yields

$$
\Delta \epsilon=\mathbf{C}: \Delta \sigma+\sum_{\mu=1}^{N} \frac{\partial \mathbf{C}}{\partial a_{\mu}}: \sigma \Delta a_{\mu}
$$


where the symbol $\Delta$ denotes small increments over a loading step. The crack radius increments $\Delta a_{\mu}$ cannot be determined from the dependence of $\mathbf{C}$ on $a_{\mu}$ (Eq. 2).

The growth of the cracks must conform to the laws of fracture mechanics. Let us assume that the cracks (actually microcracks) follow linear elastic fracture mechanics (LEFM). This means that the energy release rates must be equal to the fracture energy of the material, $G_{f}$. Strictly speaking, each of the random microcracks in the material should follow LEFM, which means that its energy release rate should be equal to $G_{f}$. To make the problem tractable, we impose the energy balance condition only in the overall, weak sense, namely that the combined energy release rate of each crack family must agree with the energy dissipation according to the material fracture energy $G_{f}$, but the individual cracks do not need to satisfy this energy balance condition. This means that we have the following $N$ conditions:

$$
\frac{\partial \Pi^{*}}{\partial a_{\mu}}=2 \pi a_{\mu} N_{\mu} G_{f} \text { for } \Delta a_{\mu}>0 \quad\left(\mu=1,2, \ldots, n_{g}\right)
$$

in which $n_{g}$ is the number of families of growing cracks, and $\Pi^{*}$ is the complementary energy of the cracked material per unit volume of the material. Repetition of subscript $\mu$ in this and subsequent equations does not imply summation unless specifically indicated. When the cracks are shortening, their faces are coming in contact, which requires no energy; therefore, for shortening cracks,

$$
\frac{\partial \Pi^{*}}{\partial a_{\mu}}=0 \text { for } \Delta a_{\mu}<0 \quad\left(\mu=n_{g}+1, \ldots, n_{s}\right)
$$

where $n_{s}$ is the number of all the families of growing and shortening cracks.

The complementary energy per unit volume of the microcracked material is expressed

$$
\Pi^{*}=\frac{1}{2} \boldsymbol{\sigma}: \mathbf{C}: \boldsymbol{\sigma}
$$

Differentiating Eq. 6 we thus obtain

$$
\frac{\partial \Pi^{*}}{\partial a_{\mu}}=\boldsymbol{\sigma}: \mathbf{C}: \frac{\partial \boldsymbol{\sigma}}{\partial a_{\mu}}+\frac{1}{2} \boldsymbol{\sigma}: \frac{\partial \mathbf{C}}{\partial a_{\mu}}: \boldsymbol{\sigma} \quad(\mu=1,2, \ldots, N)
$$

Substituting here Eq. 4 or 5 , and considering also that cracks $\mu=n_{s}+1, \ldots, N$ neither shorten nor grow (i.e., are fixed), we finally obtain the following incremental relations which must bè satisfied by the crack radius increments (positive or negative or 0 ):

$$
\begin{array}{ll}
\boldsymbol{\sigma}: \mathbf{C}: \Delta \boldsymbol{\sigma}+\left(\frac{1}{2} \boldsymbol{\sigma}: \frac{\partial \mathbf{C}}{\partial a_{\mu}}: \boldsymbol{\sigma}-2 \pi a_{\mu} n_{\mu} G_{f}\right) \Delta a_{\mu}=0 & \left(\Delta a_{\mu}>0, \mu=1, \ldots, n_{g}\right) \\
\boldsymbol{\sigma}: \mathbf{C}: \Delta \boldsymbol{\sigma}+\frac{1}{2} \boldsymbol{\sigma}: \frac{\partial \mathbf{C}}{\partial a_{\mu}}: \boldsymbol{\sigma} \Delta a_{\mu}=0 & \left(\Delta a_{\mu}<0, \mu=n_{g}+1, \ldots, n_{s}\right) \\
\Delta a_{\mu}=0 & \left(\mu=n_{s}+1, \ldots, N\right)
\end{array}
$$

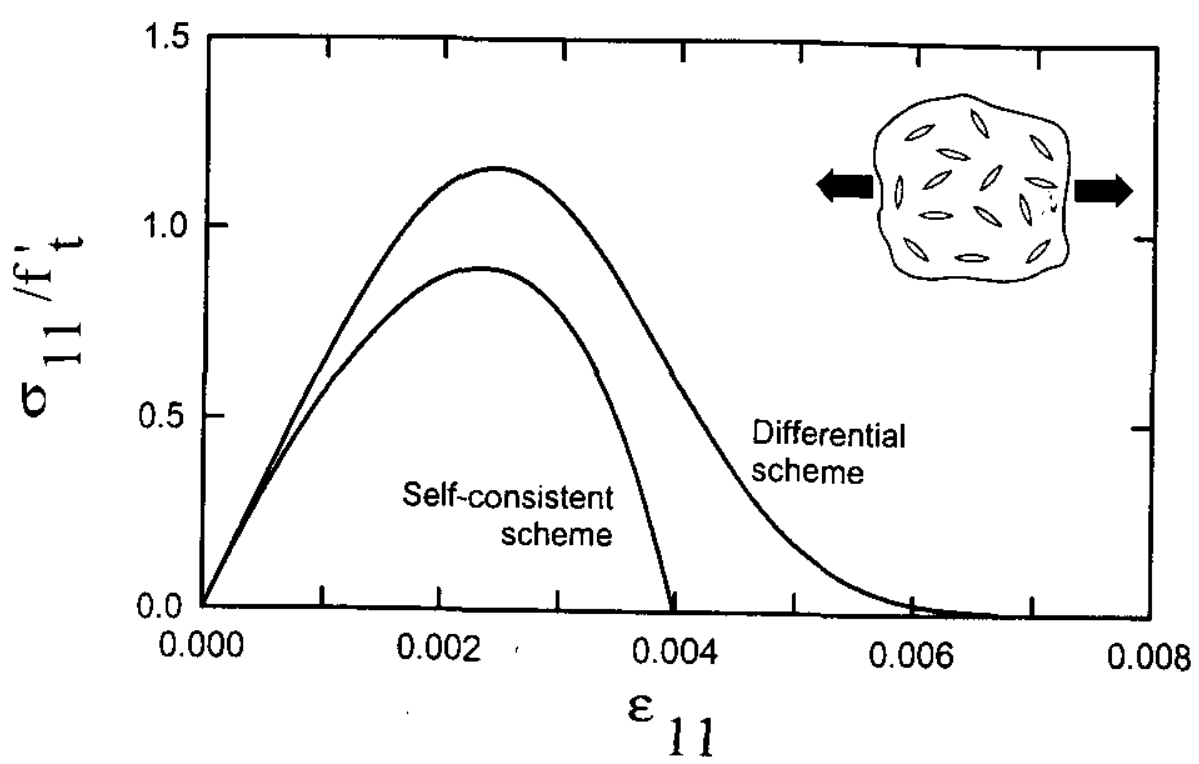

Figure 4. Uniaxial stress-strain curve for a macroscopically isotropic randomly cracked body under uniaxial tension, using the self-consistent and differential schemes.

The first equation applies to the families of growing cracks, the second to the families of shortening cracks, and the third to the families of stationary cracks.

Eqs. 3 and 8 together represent a system of $N+6$ equations for $N$ increments of crack radii and 6 increments of stress tensor components, $\Delta \sigma$. If the strain increment $\Delta \epsilon$ is prescribed, these $N+6$ unknowns can be solved from this system of equations.

The tangential stiffness tensor can be obtained by assigning a unit value to each component of $\Delta \sigma$, with the other components being 0 , and solving the corresponding stress increments for all the cases.

\section{COMMENTS ON NUMERICAL STUDIES IN PROGRESS}

Obviously, the key to the application of the present method for calculating the tangential stiffness moduli of a damaging material is the dependence of the compliance tensor on the average radii of all the crack families, as written in Eq. 2 . This problem has recently been extensively reviewed by Kachanov $[8,19]$ and considerable knowledge exists in this regard. So far only the self-consistent scheme and the differential scheme for the determination of $\mathbf{C}$ (Eq. 2) has been examined, and only for the simplest types of crack arrays.

Calculations have been run for two types of crack geometries: the first consists of a macroscopically isotropic randomly cracked body, while the second consists of a body with one single family of parallel cracks at an angle with the applied stress. In both cases, the body is subjected to a uniform stress field in tension or compression. 


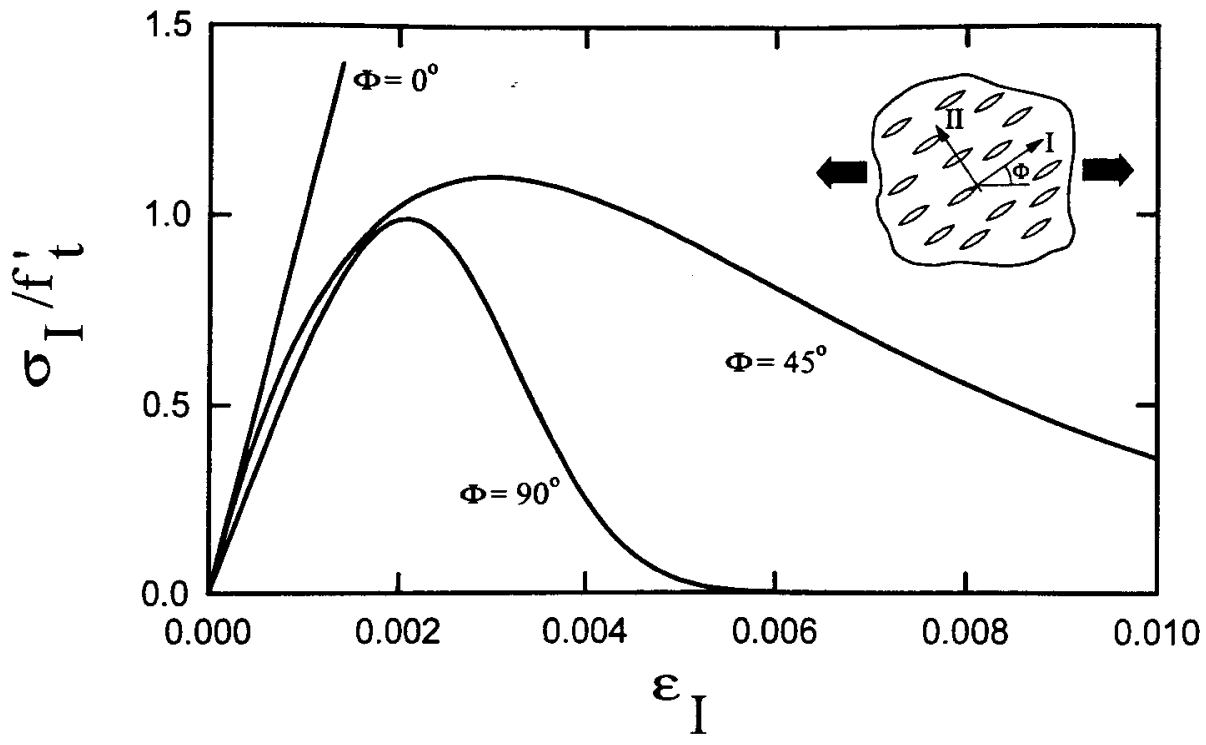

Figure 5. Results for a macroscopically orthotropic material containing one family of parallel cracks under uniaxial tension.

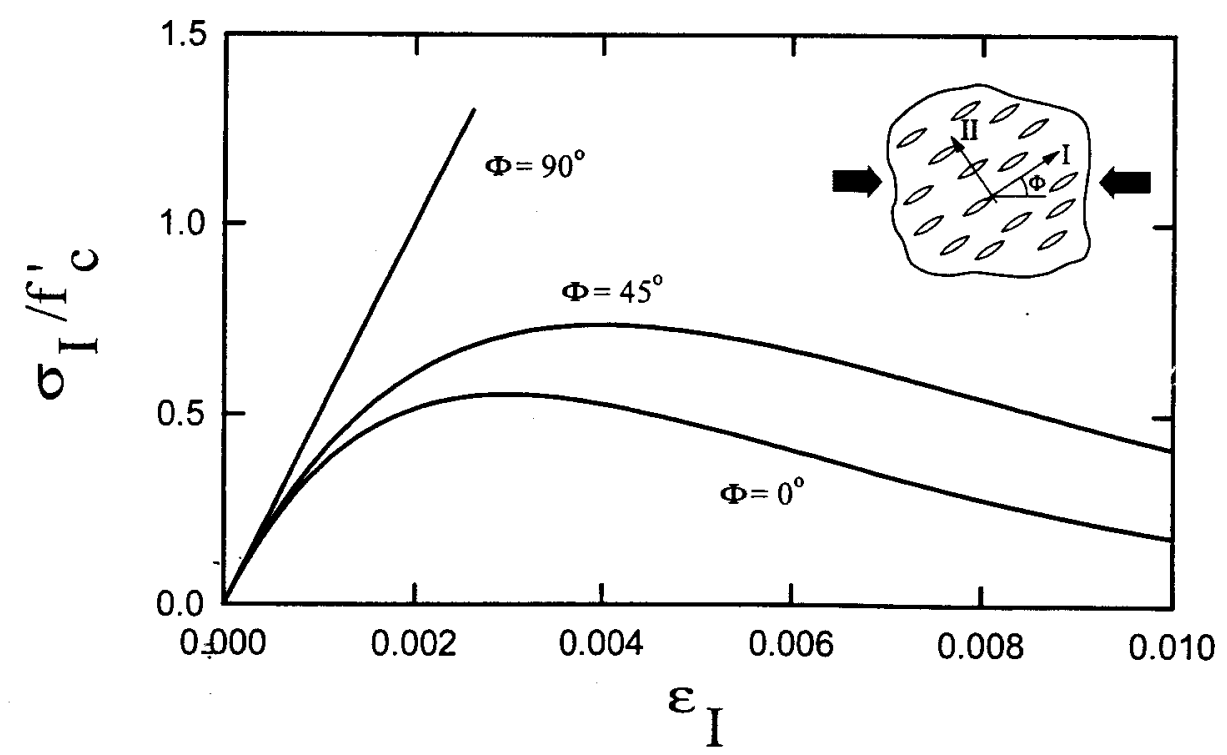

Figure 6. Results for a macroscopically orthotropic material containing one family of parallel cracks under uniaxial compression.
Fig. 4 shows the calculated uniaxial stress-strain curve for a macroscopically isotropic randomly cracked body, under uniaxial tension. The curves are shown for two calculation schemes for C: the self-consistent scheme [1] and the differential scheme (see, e.g., [18]). From this result it must be concluded that the self-consistent scheme is applicable only up to early post-peak response; after that, it predicts the stress to decrease too rapidly, at variance with test results for typical microcracking materials such as concrete. This phenomenon may be explained by the fact that the self-consistent method always yields a reduction of elastic stiffness, in terms of the crack density, that is excessive if compared with existing experimental results for microcracking materials. It has been shown $[1,23]$ that using this method, the elastic stiffness of the material vanishes for a finite value of the crack density parameter equal to $9 / 16$. The differential scheme, on the other hand, yields realistically looking stress-strain curves.

Based on this result, the self-consistent scheme appears unsuitable for predicting damage in densely microcracked materials, and the differential scheme may be recommended.

The evaluation of the compliance $\mathbf{C}$ (Eq. 2) in the calculations presented has been conducted according to a technique described in [23]. The change of compliance due to the presence of a system of cracks is evaluated by means of a symmetric second-rank crack density tensor $[4,25]$, which is a tensorial generalization of the scalar crack density, accounting for the particular crack orientations distribution. The parameters of the model are then computed by equating the results for an isotropic body (with a randomly oriented crack distribution) to the results obtained using the differential scheme.

Figs. 5 and 6 show the results for a macroscopically orthotropic material containing one family of randomly located parallel cracks. The calculated stress-strain curves are shown for uniaxial tension (Fig. 5) and uniaxial compression (Fig. 6) applied at angles $\Phi=0^{\circ}, 45^{\circ}$ and $90^{\circ}$ with the plane of the cracks. Recording the peak values of these curves, one further finds that the ratio of the compression strength to the tensile strength is about $f_{c}^{\prime} / f_{i}^{\prime}=8.6$, which is reasonable for an initially highly microcracked material such as concrete.

The numerical results presented in the preceding figures are a qualitative assessment of the model, and no attempt of data fitting has been carried out. These preliminary results show realistic trends, in good agreement with the observed behavior of the material. Further numerical studies are in progress and will be reported separately [16].

\section{CONCLUSION}

The existing results on the overall elastic constants of bodies with various systems of cracks need to be generalized to the case when the cracks grow (or shorten) during loading. In that case, the condition that the cracks remain critical, i.e., that their energy release rate remain equal to the fracture energy of the material, needs to be imposed. This condition can be written separately for several families of cracks in the material. Such conditions, together with the differentiated form of the secant elastic stress-strain relation of the microcracked material, yields a system of equations from which the stress tensor increment and the crack length increments can be solved if the strain increment tensor is given. From this, the tangential compliance tensor or tangential stiffness tensor can 
be obtained. Preliminary numerical studies reveal that the self-consistent scheme does not yield reasonable results, except for deformations slightly beyond the load peak. The differential scheme, on the other hand, yields realistically looking stress-strain curves.

For arbitrarily oriented crack systems, which render the body anisotropic, the effective elastic compliance is obtained by means of a second-order crack density tensor which embodies the particular crack distribution statistics. The differential scheme is used to evaluate the parameters of the model. Preliminary results show realistic trends resembling the experimentally observed behavior of microcracking materials. The model also predicts a ratio of compression and tensile strengths which approximately agrees with the value typical of concrete.

Acknowledgment: Partial financial support under NSF grant MSS-911447-6 to Northwestern University is gratefully acknowledged. The second author wishes to acknowledge the financial support received during his stay at Northwestern University from the AIRE program (AIRE94-12), Generalitat de Catalunya, Barcelona, Spain.

\section{REFERENCES}

[1] Budiansky, B. and O'Connell, R.J., 1976. Elastic moduli of a cracked solid. Int. J. Solids and Structures, 12:81-97.

[2] Hoenig, A., 1978. The behavior of a flat elliptical crack in an anisotropic elastic body. Int. J. Solids and Structures, 14:925-934.

[3] Hoenig, A., 1979. Elastic moduli of a non-randomly cracked body. Int. J. Solids and Structures, 15:137-154.

[4] Kachanov, M., 1980. Continuum model of medium with cracks. J. Engng. Mech Div. ASCE, 106(EM5):1039-1051.

[5] Horii, H. and Nemat-Nasser, S., 1983. Overall moduli of solids with microcracks: load-induced anisotropy. J. Mech. Phys. Solids, 31(2):155-171.

[6] Fabrikant, Y.I., 1987. Close interaction of coplanar circular cracks in an elastic medium. Acta Mechanica, 67:39-59.

[7] Ju, J.W. and Tseng, K.H., 1992. A three-dimensional statistical micromechanical theory for brittle solids with interacting microcracks. Int. J. Damage Mech., 1(1):102131.

[8] Kachanov, M., 1992. Effective elastic properties of cracked solids: critical review of some basic concepts. Applied Mechanics Review, 45(8):304-335.

[9] Mauge, C. and Kachanov, M., 1992. Interacting arbitrarily oriented cracks in anisotropic matrix. Stress intensity factors and effective moduli. Int. J. Fracture, 58:R6̄-R74.

[10] Fares, N., 1993. Effective stiffness of a periodically cracked 3-D solid. Int. J. Fracture, 62:149-162.

[11] Nemat-Nasser S. and Yu, N., 1993. Solids with periodically distributed cracks. Int J. Solids and Structures, 30(15):2071-2095.
[12] Huang, Y., Hu, K.X., and Chandra, A., 1994. A self-consistent mechanics method for solids containing inclusions and a general distribution of cracks. Acta Mechanica, 105:69-84.

[13] Ju, J.W. and Chen, T.-M., 1994. Effective elastic moduli of two-dimensional brittle solids with interacting microcracks. Part I: Basic formulations. Part II: Evolutionary damage models. J. Appl. Mech. ASME, 61:349-366.

[14] Mauge, C. and Kachanov, M., 1994. Effective elastic properties of an anisotropic material with arbitrarily oriented interacting cracks. J. Mech. Phys. Solids, 42(4):561584.

[5] Sayers, C.M. and Kachanov, M., 1995. Microcrack-induced elastic wave anisotropy of brittle rocks. J. Geophysical Research, 100(B3):4149-4156.

[16] Prat, P.C. and Bažant, Z.P., 1995. Elastic moduli of material with growing cracks. Technical Report (in preparation), Dept. of Civil Engng., Northwestern University, Evanston, IL 60208, USA.

[17] Hill, R., 1965. A self-consistent mechanics of composite materials. J. Mech. Phys. Solids, 13:213-222.

[18] Hashin, Z., 1988. The differential scheme and its application to cracked materials J. Mech. Phys. Solids, 36(6):719-734.

[19] Kachanov, M., 1993. Elastic solids with many cracks and related problems. In Hutchinson, J. and Wu, T., editors, Advances in Applied Mechanics, Vol. 30, pp. 259-445. Academic Press.

[20] Bažant, Z.P., 1994. Nonlocal damage theory based on micromechanics of crack interactions. J. Engng. Mech. ASCE, 120(3):593-617.

[21] Bažant, Z.P. and Jirásek, M., 1994. Damage nonlocality due to microcrack interactions: statistical determination of crack influence function. In Bažant, Z.P., Bittnar, Z., Jirásek, M., and Mazars, J., editors, Fracture and damage in quasi-brittle structures, pp. 3-17. E \& FN SPON.

[22] Jirásek, M. and Bažant, Z.P., 1994. Localization analysis of nonlocal model based on crack interactions. J. Engng. Mech. ASCE, 120(3):1521-1542.

[23] Sayers, C.M. and Kachanov, M., 1991. A simple technique for finding effective elastic constants of cracked solids for arbitrary crack orientation statistics. Int. J. Solids and Structures, 27(6):671-680.

[24] Kachanov, M., Tsukrov, I., and Shafiro, B., 1994. Effective moduli of solids with cavities of various shapes. Applied Mechanics Review, 47(1):S151-S174.

[25] Vakulenko, A.A. and Kachanov, M., 1971. Continuum theory of medium with cracks. Mechanics of Solids, 6:145-151. 